\title{
Epilepsia y neuroimagen. Experiencia del programa de cirugía de epilepsia del Centro Médico ABC
}

\section{Epilepsy and neuroimaging experience of the epilepsy surgery program Medical Center ABC}

\author{
José Ángel Romero Figueroa, ${ }^{*}$ Enrique de Font Réaulx, ${ }^{\ddagger}$ \\ Javier Terrazo, ${ }^{\ddagger}$ Paul Shkurovich, ${ }^{\S}$ Ernesto Ramírez Navarrete, \\ Emilio Arch Tirado," Adalberto González Astiazarán"
}

Citar como: Romero FJÁ, de Font RE, Terrazo J, Shkurovich P, Ramírez NE, Arch TE et al. Epilepsia y neuroimagen. Experiencia del programa de cirugía de epilepsia del Centro Médico ABC. An Med (Mex). 2020; 65 (3): 209-213. https://dx.doi.org/10.35366/95678

\section{RESUMEN}

Introducción: El paciente con epilepsia fármaco resistente es aquél que rebasa seis meses bajo tratamiento con uso regular de fármacos a dosis adecuadas sin alcanzar control antiepiléptico, tal situación lo hace candidato para evaluación de cirugía de epilepsia. La imagen por resonancia magnética, en conjunto con los hallazgos clínicos, es fundamental para determinar qué tipo de pacientes son candidatos para tratamiento quirúrgico. Objetivo: Resaltar la importancia de la demostración de la causa de las crisis convulsivas con la ayuda de la imagen por resonancia magnética. Material y métodos: Se realizó una investigación observacional retrospectiva evaluando a 23 pacientes del Programa de Cirugía de Epilepsia del Centro Médico ABC con datos demográficos y diagnósticos: clínico, prequirúrgico, neuroimagen y patología.

\section{ABSTRACT}

Introduction: A patient with epilepsy is considered as drug resistant, when the pharmacologic treatment lasts more than six months under correct dosage and use, and without achieving an appropriate control of the disease. This setting makes the patient a possible candidate for epileptic surgery. Magnetic resonance imaging, together with clinical findings, are essential to determine which kind of patients are good candidates for surgical treatment. Objective: To highlight the importance of magnetic resonance imaging in demonstrating the cause of seizures. Materials and methods: A retrospective observational study was performed by evaluating 23 patients included in the "Epilepsy Surgery Program" at the ABC Medical Center; the demographic, diagnostic, as well as clinical, presurgical, neuroimaging and pathology data were included. The response
* Neurorradiólogo.

‡ Neurocirujano.

$\S$ Neurología.

" Neurología Pediátrica.

"I Investigador.

Centro Neurológico, Centro Médico ABC, Ciudad de México.

Correspondencia: José Ángel Romero Figueroa Av. Carlos Graef Fernández Núm. 154, Col. Tlaxala, 05300, Alcaldía Cuajimalpa de Morelos, CDMX.

E-mail: romeroan@me.com
Recibido para publicación: 30/05/2020. Aceptado: 21/08/2020.

Abreviaturas:

$\mathrm{CE}=$ Crisis epiléptica.

$\mathrm{EFS}=$ Epilepsia focal sintomática.

EMS = Epilepsia mixta sintomática.

DCF $=$ Displasia cortical focal.

EMT = Esclerosis mesial temporal.

IRM = Imagen por resonancia magnética .

TNED = Tumor neuroepitelial disembrioplásico. 
Se evaluaron respuestas al tratamiento quirúrgico y la evolución postoperatoria. Por último, se evaluó la respuesta antiepiléptica al procedimiento quirúrgico con base en la clasificación de Engel. Resultados: Se obtuvieron resultados favorables en cuanto a la discusión clínico-neurofisiológica y de neuroimagen, resultando en la afinación de protocolos diagnósticos de condiciones epileptogénicas heterogéneas y decisiones quirúrgicas adecuadas. Conclusión: Los resultados obtenidos son congruentes con los estándares internacionales, el trabajo colaborativo de las distintas especialidades implicadas es indispensable en el campo de la cirugía de epilepsia.

Palabras clave: Epilepsia, resonancia magnética, evaluación postquirúrgica.

Nivel de evidencia: III to the surgical treatment, as well as the epileptic progression were evaluated. Finally, the antiepileptic response to the surgical procedure was evaluated with the Engel classification. Results: Favorable results were obtained according to the discussion between the clinical, neurophysiological and neuroimaging experts, which resulted in improvement of the diagnostic protocols of heterogeneous conditions as well as adequate surgical decisions. Conclusion: The results obtained are consistent with international standards, the collaborative work of the various specialties involved is essential in the field of epilepsy surgery.

Keywords: Epilepsy, magnetic resonance imaging, postsurgical evolution.

Level of evidence: III

\section{INTRODUCCIÓN}

La epilepsia es una condición anormal crónica recurrente que se expresa por crisis epilépticas. Una crisis epiléptica (CE) se origina por la aparición de actividad neuronal cerebral anormal, excesiva y sincrónica. La sintomatología de una CE es variada y depende de la localización de la zona epileptógena. Las crisis con inicio focal pueden evolucionar con otras manifestaciones dependiendo de la propagación hacia el resto de la corteza cerebral. Los pacientes pueden o no presentar alteración de la conciencia, dependiendo de si hay participación de estructuras límbicas o del tallo cerebral. ${ }^{1}$

Cuando un paciente con epilepsia es sometido a tratamiento adecuado en selección, dosis y regularidad de los fármacos indicados, y rebasa seis meses sin alcanzar control antiepiléptico, hablamos de epilepsia fármaco resistente, esto lo hace candidato a evaluación para cirugía de epilepsia. ${ }^{2}$

La imagen por resonancia magnética (IRM) ha revolucionado la evaluación prequirúrgica de la epilepsia en años recientes. ${ }^{3}$ Sin embargo, de 15 a $30 \%$ de los estudios de IRM de pacientes con epilepsia refractaria son reportados como estudios negativos, es decir, sin lesión estructural identificable. ${ }^{4}$

Un adecuado protocolo de IRM para evaluación diagnóstica de epilepsia se basa en seis secuencias que pueden identificar lesiones epileptogénicas y aportar un balance razonable entre la precisión diagnóstica y la factibilidad clínica. ${ }^{5}$ Los magnetos de $3 \mathrm{~T}$ tienen mayor poder de resolución que los magnetos de $1.5 \mathrm{~T}$ y las antenas multifase con formación en paralelo son mejores que las antenas convencionales.,7 Los métodos postprocesamiento como la imagen del tensor de difusión (ITD), entre otros, pueden ser útiles para reconocer lesiones no identificables en el análisis visual de la IRM. ${ }^{8}$

En el campo de la cirugía de epilepsia es indispensable el trabajo colaborativo del neurólogo, neurofisiólogo, neurocirujano y médico especialista en neuroimagen. La elaboración de una hipótesis para cirugía de epilepsia, su confirmación o eliminación requiere de estrecha colaboración académica de estas especialidades. Aquí presentaremos nuestra experiencia desarrollada en el Programa de Cirugía de Epilepsia ABC-JGT del Centro Médico ABC.

Objetivo: resaltar la importancia de la demostración de la causa de las crisis convulsivas con la ayuda de la IRM en pacientes con epilepsias focales, donde se incluye esclerosis mesial temporal, alteraciones vasculares, secuelas postinfecciosas, neoplasias de bajo grado y malformaciones del desarrollo cortical. ${ }^{9}$

\section{MATERIAL Y MÉTODOS}

Se evaluaron todos los pacientes de este programa iniciado en junio de 2017 a la fecha. Esta serie consta de 23 pacientes. Se presentan datos demográficos, y se analizan los diagnósticos: clínico inicial, prequirúrgico, neuroimagen y por anatomía patológica. Se evalúan respuestas y evolución epileptológica postoperatoria.

Los estudios se realizaron en equipos Siemens $3 T$ Magnetom Skyra Numaris/4, antena de cráneo de 16 canales y Phillips 1.5 Sense NV, antena de cráneo de 16 canales. El protocolo de epilepsia utilizado en nuestros pacientes incluye secuencias T1 SPGR (volumétrico 3D) para la detección de malformaciones corticales del desarrollo y la posibilidad de aplicar técnicas de postprocesamiento; secuencias T2 STIR (short tau inversion recovery) para la evaluación de 
la arquitectura hipocampal y componentes quísticos de otras lesiones); secuencias FLAIR T2 (fluid attenuated inversion recovery) en plano axial y coronal para la detección de esclerosis hipocampal, displasia cortical, tumores, inflamación y gliosis; la secuencia $\mathrm{T} 2^{10}$ eco de gradiente, o las secuencias de susceptibilidad magnética para la identificación de lesiones vasculares o calcificaciones tales como cavernomas y malformación arteriovenosa. La secuencia T2 FSE (fast spin eco) en los cerebros neonatales y maduros es mejor para mostrar lesiones corticales y el borramiento de la interfase de las sustancias gris-blanca. ${ }^{11}$

Se evaluó la respuesta antiepiléptica del procedimiento quirúrgico con base en la clasificación de Engel, ${ }_{12}$ ésta se basa en cuatro tipos de respuesta. Clase I, libre de crisis convulsivas. Clase II, crisis convulsivas esporádicas. Clase III, mejoría considerable. Clase IV, sin mejoría. A su vez, cada clase se subdivide en dos a cuatro variaciones clínicas.

\section{RESULTADOS}

Se estudiaron 23 pacientes, 14 del sexo femenino $(60.9 \%)$ y nueve del masculino $(39.1 \%)$, con un promedio de edad de $21.642 \pm 14.443$ años (media \pm desviación estándar), con un rango de 52 años (límite inferior cuatro años - límite superior 56 años). Al analizar el promedio de edad en relación al género, el femenino es de 22.071, mientras que el de los masculinos es de 21, por lo tanto, esta variable no muestra diferencias en nuestra serie.

El diagnóstico clínico inicial fue epilepsia focal sintomática (EFS) en 21 pacientes (91.3\%) y epilepsia mixta sintomática en dos pacientes $(8.7 \%)$.
En el diagnóstico clínico prequirúrgico la moda fue esclerosis mesial temporal (EMT) $\mathrm{n}=9(39.13 \%)$, probable displasia cortical focal (DCF) $n=4$ (17.39), de los cuales tres casos son del lóbulo temporal y uno del cuadrante posterior, secuelas de evento isquémico $\mathrm{n}=3$ (13\%, respectivamente), tumor glial $\mathrm{n}=$ $2(8.69 \%)$, en cinco ocasiones tuvimos diagnóstico único para las siguientes condiciones: hemimegalencefalia, epilepsia postraumática, epilepsia partialis continua, esclerosis tuberosa y trisomía 21 con disgenesias múltiples.

$\mathrm{Al}$ analizar el diagnóstico prequirúrgico por imagen se observó como hallazgo más frecuente EMT con probable patología dual en nueve casos (39.13\%), displasia cortical focal en cuatro casos (17.39\%), dos pacientes con secuelas de evento isquémico y dos pacientes con tumor glial (Figura 1), (8.69\% respectivamente), mientras que en seis diagnósticos hubo un solo caso $(4.34 \%)$.

El análisis de los resultados del diagnóstico por patología presenta nueve casos $(39.14 \%)$ que corresponden a DCF III-A, cinco casos (21.73\%) a lesión vascular, cuatro casos $(26.2 \%)$ a EMT III-A, dos casos a neoplasias, el primero con oligoastrocitoma y el segundo con TNED (8.69\%). Tres diagnósticos fueron reportados en un paciente (hemimegalencefalia, cavernoma y trisomía 21) con malformaciones múltiples.

Para evaluar la respuesta al tratamiento quirúrgico empleamos la escala de Engel. De esta forma, de 18 pacientes $(78.3 \%)$, tres $(13.0 \%)$ se encuentran en Engel I-A y dos en Engel I-B 8.7\% (Tabla 1).

Analizamos la intersección probabilística entre género, edad y la respuesta a la cirugía evaluada con la escala de Engel. La dependencia estadística entre
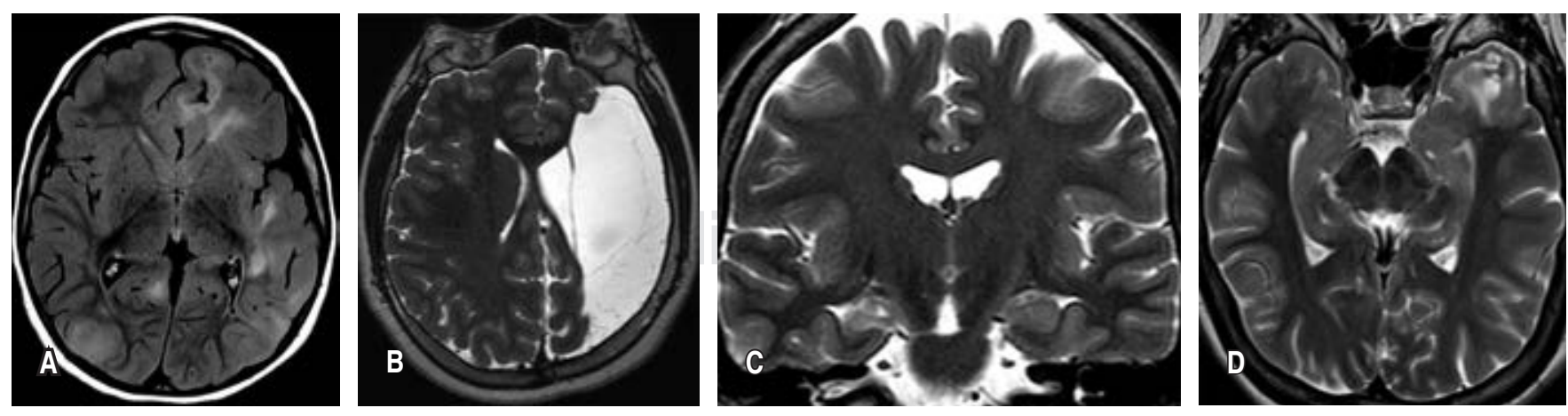

Figura 1: A) FLAIR T2 axial. Displasia cortical II-B frontal izquierda y esclerosis tuberosa. Múltiples lesiones hiperintensas córtico subcorticales (corteza expandida) en la cara dorsolateral y medial bilateral. B) T2 axial. Encefalomalacia y hemiatrofia cerebral izquierda. La imagen muestra malacia quística extensa que delinea el territorio de la arteria cerebral media izquierda como secuela de infarto probablemente perinatal. C) T2 coronal. Esclerosis mesial temporal derecha. El cuerpo del hipocampo derecho muestra tamaño disminuido (atrofia), e incremento de su señal; también se observa dilatación del receso ventricular y la fisura coroidea adyacentes (hallazgos secundarios). D) T2 axial corte con angulación al eje largo del hipocampo. Tumor neuroepitelial disembrioplásico. Lesión del polo temporal izquierdo muestra señal heterogénea de predominio hiperintenso compatible con neoplasia. 
Tabla 1: Diagnósticos por patología.

\begin{tabular}{lrrr}
\hline \multicolumn{1}{c}{ Diagnóstico } & $\mathrm{n}$ & $\%$ & $\begin{array}{c}\% \\
\text { acumulado }\end{array}$ \\
\hline Displasia cortical focal III-A & 9 & 39.13 & 39.13 \\
Secuelas de lesión vascular & 5 & 21.86 & 60.86 \\
Esclerosis mesial temporal III-A & 4 & 17.39 & 78.25 \\
Neoplasias & 2 & 8.69 & 86.94 \\
Hemimegalencefalia & 1 & 4.34 & 91.28 \\
Trisomía 21 con disgenesias múltiples & 1 & 4.34 & 95.62 \\
Angioma cavernoso & 1 & 4.34 & 99.96 \\
Total & 23 & 99.96 & \\
\hline
\end{tabular}

estas variables fue de 10/23 (43.47\%), que corresponde al género femenino Engel I-A. Es de mencionar que 9/23 (39.13\%) son menores de 40 años en el caso de los hombres, la mayor frecuencia fue también I-A $8 / 23(34.78 \%)$ con una tendencia a menores de 15 años de 5/23 (21.73\%).

Evaluamos la respuesta al tratamiento quirúrgico con base en la escala de Engel y promedio de edad. De esta forma, los pacientes con evaluación Engel I-A tienen promedio de 23.5 años, los pacientes con II-B de 22.5 y los pacientes con III-B (resultados menos favorables de la serie) con promedio de 10 años.

El análisis de la relación entre el diagnóstico clínico prequirúrgico y la clasificación de Engel y el sexo reveló que 3/23 (13.04\%) tenían un diagnóstico de epilepsia focal sintomática (EFS), posible EMT y Engel I-A, siendo todos del sexo femenino, 3/23 (13.04\%) con diagnóstico de EFS, posible DCF temporal y Engel I-A, siendo uno del sexo femenino y dos del masculino, 3/23 (13.04\%) con diagnóstico de EFS, EMT y Engel I-A, siendo dos femeninos y uno masculino, la distribución restante fue de una observación por intersección.

Con la finalidad de analizar la frecuencia entre las variables, diagnósticos por imagen precirugía, Engel y sexo se elaboró un histograma trivariado donde se observó que 6/23 (26.08\%) presentaron un diagnóstico de EMT, probable patología dual con evaluación de Engel I-A, siendo cuatro femeninos y dos masculinos, la distribución restante fue de una observación por intersección.

Se analizó la frecuencia modal entre diagnóstico de patología y clasificación de Engel, donde se observó que la frecuencia modal de 4/23 (17.39\%) fue con la evaluación de Engel I-A con EMT III-A, siendo todos del sexo femenino, en toda la distribución restante las intersecciones fueron únicas.
Para evaluar la respuesta antiepiléptica según el área quirúrgica, dividimos a los pacientes en grupo A (cirugía de lóbulo temporal) y grupo B (cirugía extratemporal). Se observó que 11/23 (47.82\%) fueron del grupo A con evaluación de Engel de I-A, de esta manera en cuanto a la muestra estudiada existe una probabilidad de $\mathrm{p}=0.4782$ de que el paciente del grupo A tenga evaluación de Engel de I-A con respecto al grupo B 7/23 (30.43\%); por último, quienes tuvieron evaluación de I-A, 3/23 (13.04\%) pertenecen al grupo B. El resultado III-A fue de 2/23 (8.68\%), que pertenecen al grupo $\mathrm{B}$.

Con la finalidad de evaluar si existía diferencia significativa en $\mathrm{p} \leq 0.05$ entre el grupo A (pacientes con resección temporal) y el grupo B (pacientes con resección extratemporal) se empleó la escala Engel. Se elaboró una tabla de contingencia, donde $\chi^{2}$ calculada fue de 5.5922, y el valor de $\chi^{2}$ tablas fue de 5.9915 , por lo que, aunque existe una diferencia, el valor obtenido fue $\mathrm{p}=0.06$.

\section{DISCUSIÓN}

Este artículo presenta los resultados del Programa de Cirugía de Epilepsia ABC, se incluyen los pacientes en forma consecutiva desde junio de 2017 hasta la actualidad. En cuanto al género, hay predominio de casos en mujeres, siendo condición opuesta en la mayoría de las series. ${ }^{14,15}$

Los rangos de edad son semejantes a los reportados por Blumcke, ${ }^{14}$ donde hay mayor número de casos adultos.

$\mathrm{Al}$ analizar los diagnósticos prequirúrgicos (clínicos y de neuroimagen) encontramos conceptos y números consistentes con algunas diferencias; sin embargo, al comparar éstos con los diagnósticos anatomopatológicos detectamos inversión en el número de pacientes con esclerosis mesial temporal y displasia cortical. De esta forma, en los diagnósticos preoperatorios la EMT fue la más frecuente y la DCF el tercer grupo por anatomopatología, esta proporción es inversa. A partir de este resultado desarrollaremos un programa de correlación diagnóstica para identificar la razón de esta divergencia.

No encontramos datos con valor predictivo para establecer pronóstico con base en la edad, sexo, diagnósticos prequirúrgicos e histopatológicos, posiblemente debido a que el tamaño de la muestra es insuficiente.

$\mathrm{Al}$ comparar resultados de las cirugías temporal y extratemporal, como en las series reportadas, ${ }^{13-15}$ los mejores resultados se observaron en la cirugía de lóbulo temporal. La significancia estadística de este 
resultado requiere una población mayor para documentar esta conclusión.

\section{CONCLUSIONES}

El Programa de Cirugía de Epilepsia del Centro Médico $\mathrm{ABC}$ ha generado resultados favorables en cuanto a discusión clínico-neurofisiológica y por neuroimagen. En años recientes se ha incrementado el uso de las técnicas avanzadas de neuroimagen multimodal. Estas modalidades incluyen la ITD, magnetoencefalografía (no disponible en nuestro país), tomografía por emisión de positrones con fluorodesoxiglucosa (PETFDG), la fusión RM/ PET-FDG, la cual es una técnica que permite la detección de anormalidades cerebrales sutiles no detectables con IRM convencional. Estas técnicas pueden guiar la resección quirúrgica en pacientes con lesiones múltiples (por ejemplo, esclerosis tuberosa) al seleccionar las lesiones que tienen mayor actividad epileptogénica, observadas como regiones de hipometabolismo durante la fase interictal en pacientes con epilepsia fármaco resistente.

De igual manera, se han afinado los protocolos diagnósticos de condiciones heterogéneas y las decisiones quirúrgicas adecuadas. Los resultados, aun siendo reducido el número de casos, están dentro de los estándares internacionales.

\section{BIBLIOGRAFÍA}

1. So E. Classifications and epidemiologic considerations of epileptic seizures and epilepsy. Neuroimaging Clin N Am. 1995; 5 (4): 513-526.

2. Berg AT, Berkovic SF, Brodie MJ, Buchhalter J, Cross JH, van Emde BW et al. Revised terminology and concepts for organization of seizures and epilepsies: Report of the ILAE
Commission on Classification and Terminology, 2005-2009. Epilepsia. 2010; 51: 676-685.

3. Baumgartner C, Koren JP, Britto-Arias M, Zoche L, Pirker SPresurgical epilepsy evaluation and epilepsy surgery (version 1; peer review: approved). F1000Research. 2019; 8: 1818.

4. Duncan JS, Winston GP, Koepp MJ, Ourselin S. Brain imaging in the assessment for epilepsy surgery. Lancet Neurol. 2016; 15 (4): 420-423.

5. Sinhu M, Duncan J, Sander J. Neuroimaging in epilepsy. Curr Opin Neurol. 2018; 31 (4): 371-378.

6. Ruber T, David B, Elger CE. MRI in epilepsy: clinical standard an evolution. Curr Opin Neurol. 2018; 31 (2): 223-231.

7. Wellmer J, Quesada CM, Rothe L, Elger CE, Bien CG et al. Proposal for a magnetic resonance imaging protocol for the detection of epileptogenic lesions at early outpatient states. Epilepsia. 2013; 45 (11): 1977-1987.

8. Rabaud C, Widjaja E. Development and Dysgenesis of the Cerebral Cortex: Malformations of Cortical Development. Neuoimag Clin N Am. 2011; 21: 483-543.

9. Cendes F, Theodore WH, Brinkmann BH, Sulc V, Cascino GD. Neuroimaging of epilepsy. Handb Clin Neurol. 2016; 136: 985-1014. doi: 10.1016/B978-0-444-53486-6.00051-X PMCID: PMC5256664 NIHMSID: NIHMS841201 PMID: 27430454.

10. Widjaja E, Raybaud C. Advances in neuromaging in patients with epilepsy. Neurosurg Focus 2008; 25: E3.

11. Kotikalapudi R, Martin P, Marquetand J, Lindig T, Bender B, Focke NK. Systematic assessment of multispectral voxelbased morphometry in previously MRI- negative focal epilepsy. AJNR Am J Neuroradiol. 2018; 39 (11): 2014-2021.

12. Engel J, Jr, Van Ness P, Rasmussen TB, Ojemann LM. Outcome with respect to epileptic seizures. In: Engel J Jr, editor. Surgical Treatment of the Epilepsies. 2nd ed. New York: Raven Press; 1993. pp. 609-621.

13. Dodrill C et al. Proposal for a new classification of outcome with respect to epileptic seizures following epilepsy surgery. In: Epilepsy surgery. 2nd ed. Lippincott Williams and Wilkins, 2001, pp. 987-990.

14. Blumcke I, Spreafico R, Haaker G, Coras R, Kobow K, Bien $\mathrm{CG}$ et al. Histopathological findings in brain tissue obtained during epilepsy surgery. N Engl J Med. 2017; 377: 1648-1656.

15. Lüders HO. Protocols and outcome statistics from epilepsy surgery centers. In: Tribal. Epilepsy surgery. 2nd ed. Lippincott Williams and Wilkins, 2001, p. 977. 